\title{
Harmonic Distortion and Oscillatory Voltages and the Role of Negative Impedance
}

\author{
P.J.M. Heskes, J.M.A. Myrzik, Member, IEEE and W.L. Kling, Member, IEEE
}

\begin{abstract}
This paper focuses on two power quality effects that are noticed with power electronic appliances. One effect is a harmonic oscillation by inverters of photo voltaic systems because they bring a high parallel capacitance to the grid and negative output impedance. The other effect is a sub-harmonic oscillation due to the negative differential impedance of constant power loads.
\end{abstract}

This paper discusses both effects and proposes counter measures to mitigate these effects. For harmonic oscillations due to photo voltaic inverters, an extra feature for these inverters is discussed. For the sub-harmonic oscillatory voltages due to appliances with negative differential impedances, a solution can be found in the voltage control systems of the power generators connected to the network.

Index Terms--Constant Power Load, Harmonic Interaction, Negative Differential Impedance, Negative Impedance, Oscillatory Grid Voltage, Photo Voltaic Inverter, Power Quality.

\section{INTRODUCTION}

$\mathrm{T}$ ODAY's appliances that are equipped with a power electronic converter can bring a negative differential impedance behaviour, which is caused by the converter control system. This kind of impedance can bring an oscillatory grid voltage in a frequency range below the fundamental frequency [01], [02]. Other voltage instability effects in the grid are noticed with inverters for photo voltaic systems. They can bring negative absolute impedance to the grid in the harmonic frequency range. This kind of impedance can cause resonances in a frequency range above the fundamental frequency, which can provoke harmonic voltages in local distribution grids [03].

The work presented in this paper is part of the research project 'Voltage quality in future infrastructures' ('Kwaliteit van de spanning in toekomstige infrastructuren (KTI)' in Dutch), sponsored by the Ministry of Economics Affairs of the Netherlands.

P.J.M. Heskes, is with the Energy Research Centre of the Netherlands $(\mathrm{ECN})$ at the Intelligent Energy Management group and as a Ph.D. student at the Electrical Power Systems group of the Eindhoven University of Technology. Email: heskes@ecn.nl

J.M.A. Myrzik, is professor at the Dortmund University of Technology in the field of energy efficiency. Email: johanna.myrzik@tu-dortmund.de

W.L. Kling is professor at the Eindhoven University of Technology, faculty of Electrical Engineering, in the field of sustainable energy systems, and chair of the Electrical Power Systems group. Email: w.l.kling@tue.nl
This paper focuses on both effects and gives a description of the role of the network impedance herein. A simplified model is given to illustrate the harmonic interaction inside a local distribution system. Counter measures to control both effects are proposed. For harmonic distortion in local distribution systems due to resonances an active approach is discussed, that makes use of a combination of extra features (ancillary services) of power electronic inverters of distributed generators. For the low frequency oscillatory voltages caused by negative differential impedances, a solution can be found in voltage control systems of the involved generators.

An oscillatory grid voltage due to the negative differential impedance of constant power loads is a phenomenon that already showed up in small micro grids like electricity systems on board of ships, where generators became instable because their control systems were responding on a large share of constant power loads [04]. Beside the rapidly growing numbers of constant power loads, today's ideas of future electricity systems propagate the possibility of isolating local distribution systems in critical situations [05]. The chance that a constant power load takes the overhand is much higher in these small isolated systems; therefore this form of instability will firstly become a problem in small grids. However it might also show up in larger electricity grids when the constant power load share continues to grow.

\section{IMPEDANCE AS TRANSFER FUNCTION}

In general it can be said that harmonic currents are transferred into harmonic voltages via network impedances. In a local distribution system, all cable parts and the transformer have impedance. Especially for the harmonic frequency range the dominating impedance in this is the leakage inductance of the transformer, therefore in a more simplified model of a distribution system, the cable impedances can be neglected. With this assumption, the network can be simplified to the model of Figure 1, just for understanding the system of harmonic interaction.

With this model it easily can be seen that voltage drop over the network impedance will affect the voltage of all the loads connected to the Point of Common Coupling (PCC). This counts for the fundamental as well as for harmonics. The load in the model is a non-linear load and modeled as a linear load with harmonic current sources in parallel [06]; only one harmonic source is depicted. 


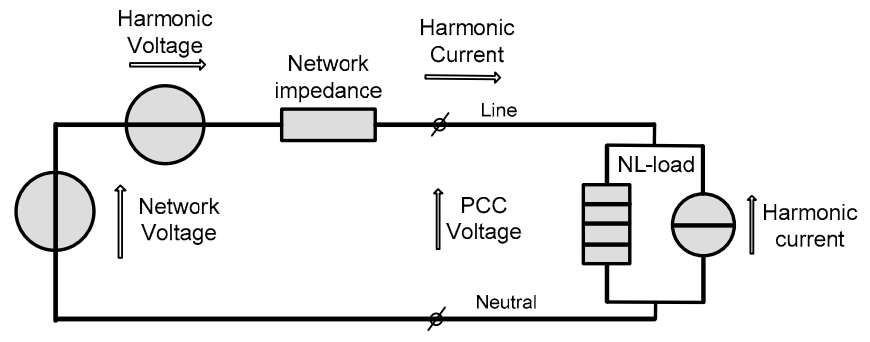

Figure 1, A simplified distribution network model.

Harmonic currents from non-linear loads will distribute itself over all impedances in the network, and the lowest impedance path will have most of the share. The part of the harmonic currents that is flowing through series impedances, like the network impedance of Figure 1, will be the main cause for the transfer into harmonic voltages at the PCC.

Harmonics from non-linear loads outside the network can be modeled as an added voltage source in series with the fundamental voltage [07] (see Figure 1). The network impedance in this figure is the distribution transformer impedance plus the impedance of the next higher network where the disturbing load is located; the latter impedance is lower in magnitude then that of the distribution transformer. In practice this so called background distortion can be significant with large non-linear loads.

In case of background voltage distortion on the fundamental, all loads in the low voltage network will draw current from this harmonic voltage source. This current then will flow through the network impedance, i.e. the distribution transformer, which can bring a number of unwanted effects to this component.

Impedance can be seen as a transfer function from current to voltage. To get more insight in possible voltage instability with this, a general equation for a linear transfer function in the Laplace domain is given in (1).

$$
H(s)=k \frac{\prod_{p=1}^{m}\left(s-z_{p}\right)}{\prod_{q=1}^{n}\left(s-p_{q}\right)}
$$

Wherein $s=\lambda+j \omega$ is the complex frequency variable, $\mathrm{z}_{\mathrm{p}}$ the zeros and $\mathrm{p}_{\mathrm{q}}$ the poles of the transfer function. Due to the fact that physical systems cannot increase the response to infinite with a frequency rising to infinite, the order of the numerator must be equal or less then that of the denominator, so $n \geq m$ in (1).

Poles of this impedance transfer function that lie in the right half-plane of its PZ-map indicate instability. The voltage instability effect that can show up from this transfer function $H(s)$, is that the transition from one output state to a new state, will not be damped to zero, but starts with an oscillation. A remarkable fact is that if the transfer function is taken the opposite way, from voltage to current, impedance will be turned into admittance, and poles will turn into zeros and the other way round, zeros will turn into poles. With this a stable system can become unstable. An example of this is the fluorescent lamp, that cannot be driven in a stable way directly (without a ballast) from a voltage source, but on the other hand can be driven in a stable way directly from a current source [08].

\section{A. Negative impedance}

Impedance is called to be negative, when the real part of the complex impedance is negative. Assume that there are appliances in the grid that show a negative impedance value, then these particular impedances can reduce the damping in the system because poles are shifted towards the positive real half-plane of the PZ-map. If the number of this kind of impedances rises significantly, poor damped resonances might occur. This phenomena can show up when the grid voltage level goes from one state to a new state, as can be seen further in this paper.

\section{ABSOLUTE AND DIFFERENTIAL IMPEDANCE}

Impedance can turn out to be absolute or differential, depending on the way the system is stimulated. The absolute impedance of a grid connected appliance is the operating point voltage, divided by the current through it. The differential impedance is the impedance that counts for deviations round an operating point. In practice the operating point is the grid fundamental voltage and the deviations can be a slow variation like a flicker distortion, a sub-harmonic or an oscillation.

Non-linear grid connected appliances can present a different impedance for each operating point. Appliances that can be specified with only one impedance figure for all operating points are pure linear components without saturation effects. For example, a pure resistor has the same impedance for all frequencies in all operating points, where the absolute and differential impedance is just the resistor value. Generally this is not the case; even an incandescent lamp is not linear due to the temperature dependence of the filament resistance. This temperature effect is a slow process; therefore its differential impedance differs from the absolute impedance at slow varying voltage levels.

Loads or sources connected to the grid with power electronic converters have the potential to have negative absolute, as well as negative differential impedance, for certain frequencies. This can lead to voltage instability [01], [09].

\section{NeGATIVE ABSOLUTE IMPEDANCE}

The V-I plot of a negative absolute impedance is shown in Figure 2, however this cannot exist with a single physical component, also an energy source is required. Inverters for Distributed Energy Resources (DER) can show a negative absolute impedance in a certain frequency range [10]. 


\section{A. Inverters}

Inverters can have a negative absolute output impedance in the harmonic frequency range that can reduce damping in the grid and beside this, they can cause resonances for even lower then the $25^{\text {th }}$ harmonic, due to the high parallel capacitance at the feeders. The negative absolute impedance in the harmonic frequency range is an effect that depends on the inverters control system [09].

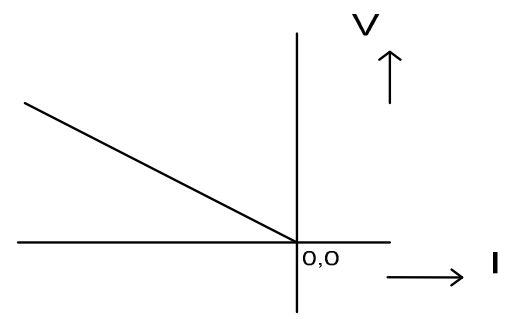

Figure 2, A negative absolute impedance.

\section{1) Parallel capacitance and grid resonances}

Because today's modern home appliances and small inverters for DER bring in more and more parallel capacitances to the grid, the effect on the total grid impedance in the low voltage distribution grid is significant. Although these capacitances are small for home appliances, due to the large and still increasing numbers, the total parallel capacitance in distribution grids can rise to a high level. In today's practice the EMI filter capacitances of DER inverters are much higher than those of modern home appliances of comparable power size. Installing a DER inverter of $2 \mathrm{~kW}$ or more in a dwelling, can triple the total capacitance at the Point of Connection $(\mathrm{PoC})$.

Parallel capacitances and inductive grid impedances can come into resonance. This resonance can let the impedance around the resonance frequency, rise to a much higher level. In this way harmonic currents that lie in the same frequency range can be transferred into problematic high harmonic voltages. These resonances are a real threat for power quality. Beside the resonance fenomenon, an inverter might also reduce the damping of this resonance by bringing in a negative impedance [03].

\section{B. Laboratory tests on an inverter load}

To estimate the potential to reduce damping in the grid by a small single-phase PV inverter, laboratory measurements where done that show the real part of the complex conductance. The first 50 harmonics were examined on a small single-phase PV inverter with an output power less then $300 \mathrm{~W}$. The inverter is equipped with fast switching power transistors and a high frequency ferrite transformer.

In Figure 3, the measured $\mathrm{Gn} / \mathrm{Gref}$ is presented for a number of harmonics. $\mathrm{Gn}$ is the real part of the harmonic conductance and Gref is a constant to normalise the conductance. Gref depends on the nominal inverter power.

In Figure 3 it can be seen that $\mathrm{Gn} / \mathrm{Gref}$ is negative up to the 25 th harmonic, this means that the inverter reduces damping in the grid which can provoke oscillations in this frequency range. $\mathrm{Gn} / \mathrm{Gref}$ is strongly positive from the 25 th up to the 50th harmonic; this means that the inverter brings extra damping to the grid in this frequency range, compared to a linear load.

This behaviour strongly depends on the inverter's control system and is not a common practice; another type of inverter can show a different behaviour [11].

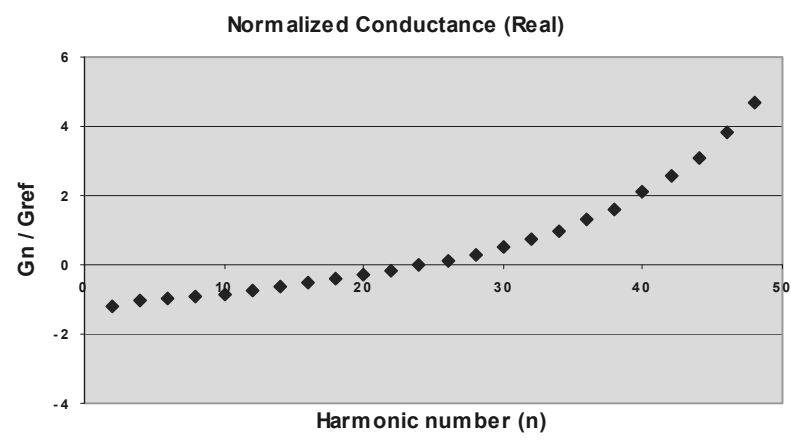

Figure 3, Real part of the inverters conductance (normalized).

\section{Harmonic voltage distortion due to resonances}

An example of a practical situation is taken from the Bronsbergen holiday park in Zutphen, the Netherlands [12]. The situation there is as follows:

- one $400 \mathrm{kVA}$ transformer

- $108 \mathrm{PV}$ inverters of $2.5 \mathrm{~kW}$

- 210 cottages.

The peak of the parallel resonance can be expected at the $12^{\text {th }}$ harmonic, this can be calculated with the estimated figures below:

- $400 \mathrm{kVA}$ transformer leakage ind. $\quad 50 \mu \mathrm{H}$

- 108 pcs. $2.5 \mathrm{~kW}$ PV inverters cap. $\quad 810 \mu \mathrm{F}$

- 210 cottages parallel cap. $\quad 630 \mu \mathrm{F}$.

Figure 4 gives a measurement result of the voltage of one of the substation feeders of the Bronsbergen holiday park. Two fairly high harmonic levels can be noticed at the $11^{\text {th }}$ and $13^{\text {th }}$ harmonic, a resonance according to the estimation could be the cause of this.

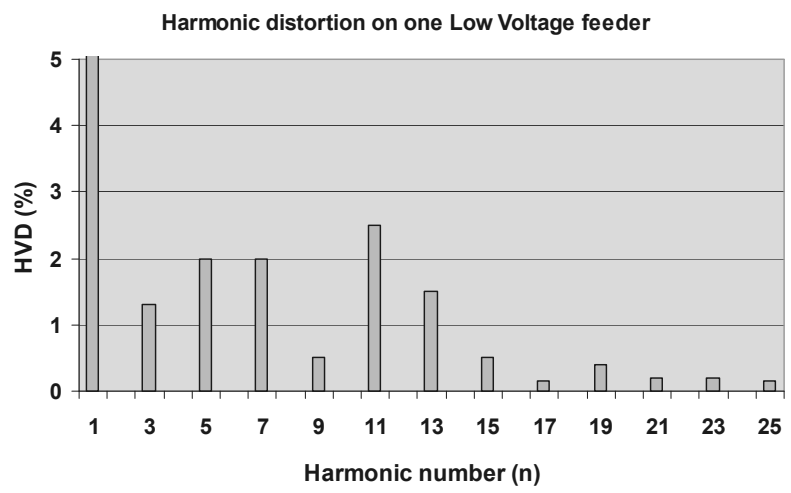

Figure 4, Harmonic voltages of one of the substation feeders in the Bronsbergen holiday park. 


\section{Reduction of grid resonances and harmonics}

In this investigation, the proposed reduction of grid resonances and harmonics is a combination of two measures, firstly shifting the resonance out of the harmonic area of concern; that is below the $40^{\text {th }}$ harmonic, and secondly damp the resonance peak to a lower level. The second measure will also lead down harmonic currents.

\section{1) Shifting of resonances}

Shifting resonances is a method to avoid resonances in the frequency area of concern. In situations where distribution networks are not having large capacitances in parallel to the load, the possible resonances are in general in higher frequency ranges than the area of concern. In this higher frequency range harmonics will not propagate far in the network, because of the damping effects of cables and transformers [13]. The solution lays thus in reducing the high parallel capacitance of inverters for DER. In Figure 5 the effect of a reduced capacitance can be seen on a calculated Bode impedance plot.

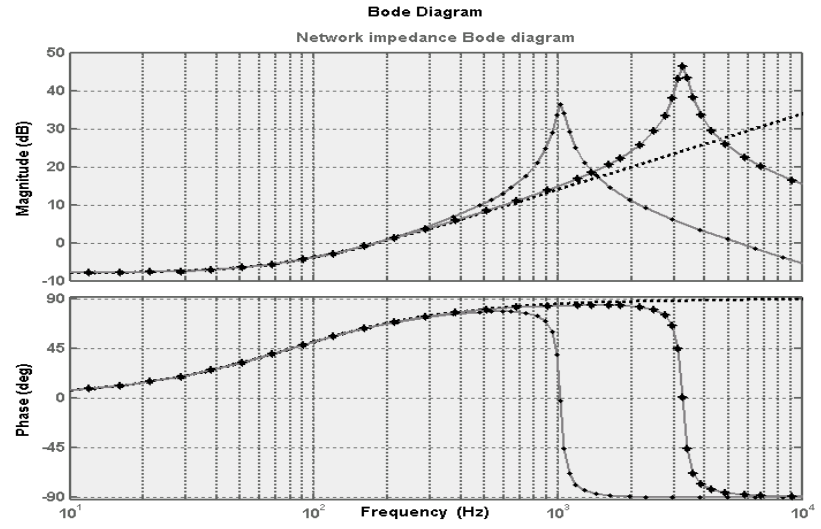

Figure 5, Bode impedance plot with a parallel resonance, dotted line: Unloaded network, line with dots: High par. cap. inverter load, line with asterisk: Low par. cap. inverter load.

To reduce the parallel capacitance, an extra control system can be added to the inverters prime control system, that virtually reduces this capacitance for all frequencies lower then a certain harmonic number [14]. With this, the EMI performance of the output filter is not affected.

\section{2) Damping of resonances}

The second measure for the reduction of resonances is to add an extra control loop to the inverter which gives the inverter a resistive behavior for the harmonic frequency range. This will bring extra damping to resonances in the network [15], [16]. As indicated in Figure 6, implementing the combination of both methods can be very effective for resonances.

\section{3) Damping of harmonics}

Beside the damping of resonances the inverters virtual resistive behavior brings, it also leads down harmonic currents from non-linear loads and with that reduce harmonic voltages at the PCC. There is however a contradiction with the needed measure for harmonics from outside the distribution network, the so called background harmonics, therefore the value of this virtual resistor must be limited to restrict harmonic currents through the distribution transformer, cables and/or lines [07], [17].

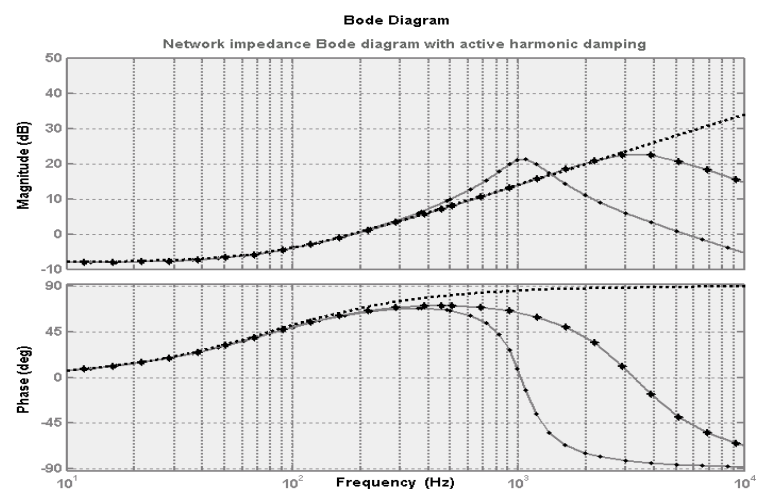

Figure 6, Bode impedance plot with a damped parallel resonance.

\section{NEGATIVE DIFFERENTIAL IMPEDANCE}

Loads with power electronic converters that draw a constant power from the grid at various grid voltage levels, are constant power loads. These kind of loads have the potential to show a negative differential impedance for certain frequencies.

\section{A. Constant Power Load}

Just as with non-linear loads in general, the impedance of a constant power load depends on the applied voltage. In practice, a lot of home appliances are loads with $\mathrm{AC}$ to DC converters inside, feeding an internal part that works on a constant direct voltage. The control system of these AC to DC converters regulates the direct voltage $U_{\mathrm{dc}}$ in such a way that the voltage is independent of the applied grid voltage $\mathrm{U}_{\mathrm{ac}}$. This implies that the AC power remains constant if the grid voltage varies. If the grid voltage increases, the grid current will decrease and also the other way round, if the grid voltage decreases, the grid current will increase. This behaviour brings negative differential impedance and this can lead to voltage instability [01].

In Figure 7, the $\mathrm{V}_{\text {RMS }} \mathrm{I}_{\mathrm{RMS}}$ curves of two typical loads are given. The left side figure could be that of an incandescent lamp, where the dashed line is the absolute impedance for a certain operating point that depends on the applied RMS voltage, and the straight solid line the positive differential impedance in that operating point. The right side figure could be that of a power supply, which shows a negative differential impedance.
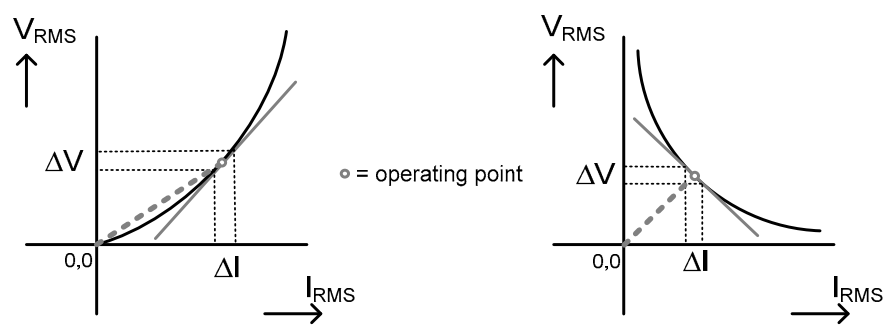

Figure $7, \mathrm{~V}_{\text {RMS }} \mathrm{I}_{\mathrm{RMS}}$ curves of two typical loads. 
To show the negative differential impedance effect, the operating point of a constant power load must be changed. This change happens when the amplitude of the grid voltage is fluctuating slowly, in a way that the appliance is able to follow this variation with a changing operating point. This can happen when a low frequency distortion voltage modulates the grid fundamental voltage.

Formula 4 below gives the situation where the fundamental voltage $u_{f}$ (2) is modulated with a small, low frequency distortion voltage $u_{d}$ (3):

$$
\begin{aligned}
& u_{f}=\hat{u}_{f} \cos \left(\omega_{f} t\right) \\
& u_{d}=\hat{u}_{d} \cos \left(\omega_{d} t\right)
\end{aligned}
$$

Then the total modulated grid voltage $u_{\text {mod }}$ is:

$$
u_{\text {mod }}=\left(\hat{u}_{f}+\hat{u}_{d} \cos \left(\omega_{d} t\right)\right) \cos \left(\omega_{f} t\right)
$$

From (4) can easily be seen that due to the modulation, the amplitude of the applied fundamental voltage on a constant power load can vary in time. Modulation gives a slow varying amplitude when the frequency of $u_{d}$ is much lower than the frequency of $u_{f}$. This means that the operating point of a constant power load can be changed and that the negative differential impedance effect can show up.

Based on data from laboratory measurements calculations to find the differential impedance are done with the help of Matlab/Simulink software and Discrete Fourier Transformation (DFT). These calculations count for small signals, because the differential impedance only can be seen as linear for small signals around an operation point.

\section{B. Laboratory tests on a constant power load}

To measure the differential impedance of a home appliance, a PC + LCD screen load is brought to a laboratory and subjected to a modulated $230 \mathrm{~V} / 50 \mathrm{~Hz}$ single-phase grid voltage from a grid simulator. A drawing of this set-up is given in Figure 8.

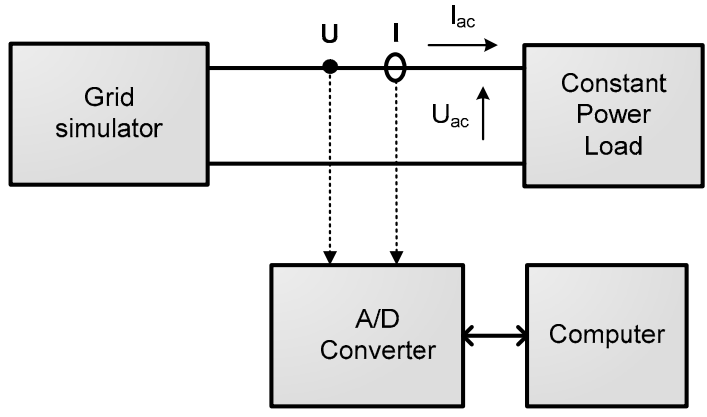

Figure 8, Measurement set-up on a constant power load.

With this set-up time series of the sampled input voltage and current are stored and analyzed via DFT in a computer with Matlab/Simulink, to calculate the differential impedance in the frequency domain. The grid voltage is modulated with a series of frequency from $0.02 \mathrm{~Hz}$ to $5 \mathrm{~Hz}$. A Polar plot of the calculated impedance of this system is shown in Figure 9.

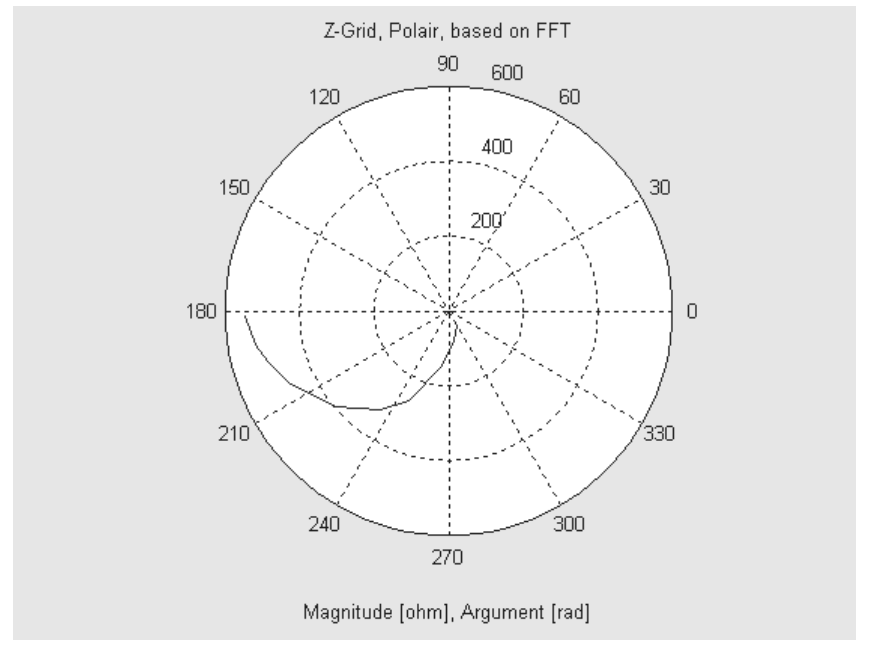

Figure 9, Polar plot of measured negative differential impedance.

Figure 9 points at a system that starts from pure resistive negative differential impedance at frequencies below $0.02 \mathrm{~Hz}$, towards a more capacitive-resistive system at higher frequencies. Although the polar plot does not show frequency on a scale, the differential impedance turns from negative to positive at about $4 \mathrm{~Hz}$. So if this negative differential impedance brings oscillations in the grid voltage, they are expected below $4 \mathrm{~Hz}$.

\section{Oscillatory voltages}

In this section an oscillatory grid voltage is simulated with the help of a Matlab/Simulink computer model. The model is build out of a generator feeding in the first place an inductive load and a resistive pre-load (see Figure 10). In the second place, after switching, the resistive pre-load is removed from the network and replaced by a combination of a resistive load and a constant power load. With this simulated set-up, the effect of various constant power load shares on voltage oscillation is analyzed.

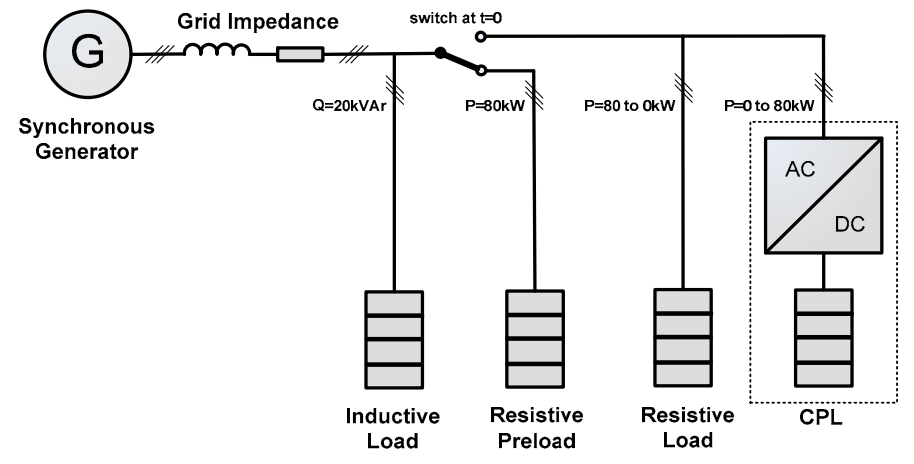

Figure 10, Generator-load model.

For the generator, a detailed model of a Diesel-Generator combination from the Matlab/SimPowerSystems library was used in the first place. To speed-up the simulations, this part was replaced by a more simplified dedicated model, with comparable results in this field of interest. The chosen inertia constant has a value of $\mathrm{H}=1.5 \mathrm{~s}$. Further the nominal generator power equals $120 \mathrm{kVA}$. The generator speed is controlled by a governor system and the generator output voltage is controlled by a field winding control system. 
The constant power load has an internal AC/DC converter loaded with a resistor at the DC output voltage. Although this converter is a three-phase type, an internal buffer capacitor of $3000 \mu \mathrm{F}$ was used in the model. The maximum power of this load is $80 \mathrm{~kW}$. The power of the constant power load is adjusted by changing the load on the DC output side of the converter. The loads applied to the generator do not exceed the generators maximum power level. With this, voltage collapse is not noticed, only oscillatory voltages.

In Figure 11, the line RMS Voltage as function of time is plotted with different load situations, combined to a threedimensional plot. The generator is loaded in all cases with $20 \mathrm{kVAr}$ inductance. Beside this, $80 \mathrm{~kW}$ active power is drawn from the generator, by a mixture of resistive load and constant power load, so the total active power equals $80 \mathrm{~kW}$ for each combination. The expressed percentage of the constant power load is referred to the total load of $80 \mathrm{~kW}$, so $50 \%$ constant power load means that the grid is loaded with $40 \mathrm{~kW}$ constant power load and $40 \mathrm{~kW}$ resistive load.

Before the starting point time $=0$, the grid is in a stable condition, preloaded with $80 \mathrm{~kW}$ resistive load and $20 \mathrm{kVAr}$ inductive load. The resistive preload is switched off at time $=0$ at the first current zero-crossing. Directly hereafter at a zero-crossing of voltage, the variable resistive part from the linear load and the constant power load are switched on. The small delay results in a step response, which shows the damping of the system.

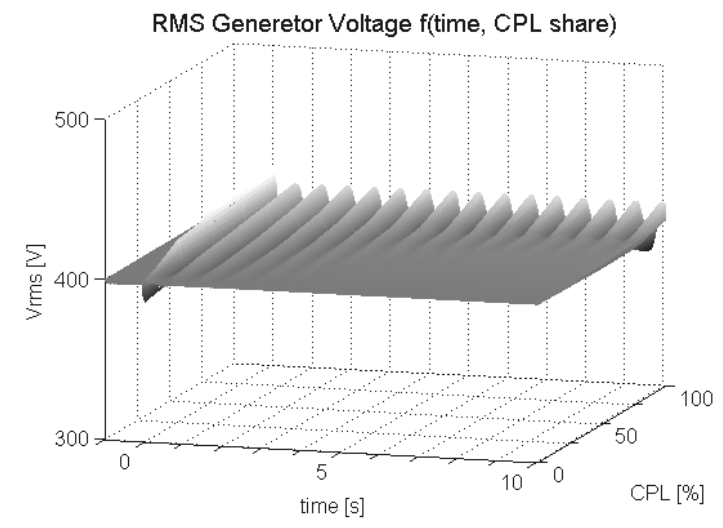

Figure 11, Effect of a constant power load share in the Generator-Load Model.

As can be noticed from Figure 11, at a constant power load share of about $85 \%$ and more, the voltage in this simulated set-up remains oscillating.

\section{Reduction of oscillatory voltages}

In the simulation of the previous paragraph, the voltage control settings of the generator model were default. The control system performed well without the presence of a constant power load in the model. But these simulations have shown that the generator voltage control system is strongly affected by the presence of a constant power load. Therefore the performance setting of the generator voltage control system is adjusted in this paragraph, to see the effect on grid voltage oscillations. The voltage control performance is expressed in its frequency bandwidth. A bandwidth of 1PU is the default value, used in the simulations of the previous paragraph, reducing this figure means a reduction in the voltage control speed.

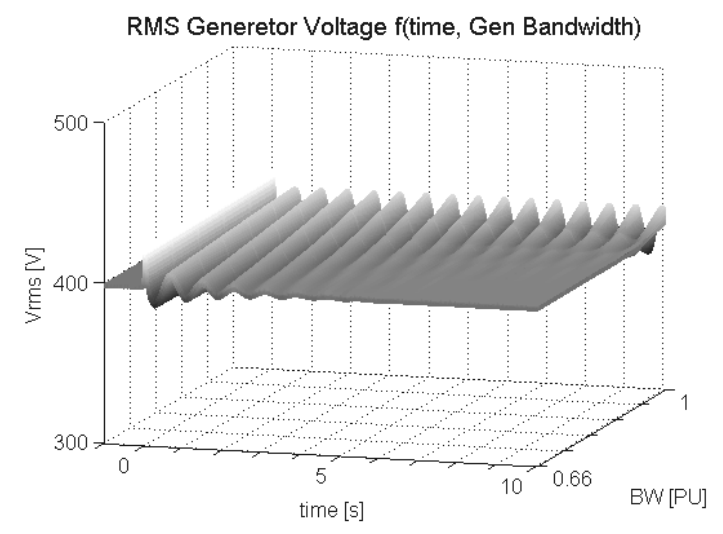

Figure 12, The effect of the generator voltage control bandwidth, while loaded with $100 \%$ constant power load.

As can be noticed in Figure 12, the generator voltage control bandwidth in this simulated set-up needs to be reduced only slightly to free the system from poorly damped oscillatory voltages. Most important here is the increase in gain and phase margin of the generators voltage control system.

\section{CONCLUSIONS}

The combination of virtual reduction of parallel capacitances and virtual harmonic resistive damping of the inverters for DER is very effective to reduce resonances caused by the implementation of large numbers of inverters and modern appliances with power electronic converters. The effect can be on a large frequency range that includes a number of harmonics. The involved ancillary control system needs no estimating of the actual level of harmonics in the network. Beside this, there is no fear for instabilities involved with this system. Because the damping resistance is virtual, the energy involved is limited to small losses in the inverter. Another advantage of being virtual is that the only effort to be taken is an extension of the control system of the inverter, with this, costs can be kept minimal.

A disadvantage is that there is a contradiction in the needed measure for background harmonics, harmonics from the next higher network. Therefore the reduction must be limited to avoid wrong compensation, resulting in excessive harmonic currents through the distribution transformer and lines. However, a combination with a series active filter on substation level can solve this problem.

From simulation with Matlab/Simulink and laboratory measurements, the following conclusions can be made concerning oscillatory voltages for single and three-phase appliances with a significant buffer capacitor and constant power load behaviour. Interaction of the negative differential impedance of appliances and the voltage control 
systems of generators can cause low frequency oscillatory voltages, especially in small isolated grids or distribution areas that operate in island mode. This is because the energy supply is small and easily affected by a constant power load share that takes the overhand. But it can also show up in larger electricity networks when the constant power load share remains growing at various power levels.

A solution for this problem can be found in changing the parameters of voltage control systems of generators to achieve more gain and phase margin.

\section{REFERENCES}

[01] A. Emadi, Modeling of Power Electronic Loads in AC Distribution Systems, USA, Transactions on Industrial Electronics, Vol. 51, No.5, 2004.

[02] M. Molinas et al, Norwegian University of Science and Technology, Trondheim, D. Moltoni et al, Politecnico di Milano, Italy, "Investigation on the Role of Power Electronic Controlled Constant Power Loads for Voltage Support in Distributed AC systems", 39th IEEE PESC08, Rhodos, Greece, 2008.

[03] J.H.R. Enslin; W.T.J. Hulshorst; A.M.S. Atmadji (KEMA, The Netherlands), P.J.M. Heskes; A. Kotsopoulos (ECN, The Netherlands), J.F.G. Cobben; P. Van der Sluijs (NUON, The Netherlands), "Harmonic Interaction Between Large Numbers of Photovoltaic Inverters and the Distribution Network", IEEE Transactions on Power Electr. Vol.: 19, No. 6, Nov. 2004.

[04] C. Rivetta, G. A. Williamson, and A. Emadi, "Constant power loads and negative impedance instability in sea and undersea vehicles" in Proc. 2005 IEEE Electric Ship technologies Symposium, Philadelphia, PA, July 2005.

[05] Raphael Caire et al, IDEA, France; "High-level Specification of the Functionalities for Novel Electricity Distribution Grid Control", Deliverable D2.1 of the INTEGRAL EU-Project, FP6-038576; 1709-2008.

[06] C. Collombet, J.M. Lupin, J. Schonek, (Schneider Electric, France), Harmonic disturbances in networks and their treatment, Cahier technique no. 152 .

[07] IEEE 519-1992, Recommended Practices and requirements for harmonic control in Electr. Power Systems, (1992), IEEE.

[08] E. Deng 1), S. Cuk 2), Negative Incremental Impedance and Stability of Fluorescent Lamps, 1) GE Corporate R\&D, New York, USA, 2) California Institute of Technology, California, USA, IEEE, 1997.

[09] P.J.M. Heskes, J.F.G. Cobben, H.H.C. de Moor "Harmonic distortion in residential areas due to large scale PV implementation is predictable" International Journal of DER, Vol. 1, No. 1, 2005.

[10] P.J.M. Heskes, P.M. Rooij (ECN, The Netherlands), J.F.G. Cobben (TU/e, The Netherlands), H.E. Oldenkamp (OKE-services, The Netherlands), "Estimation of the potential to pollute the electricity network with harmonics due to the use of small micro generators with inverters" ECN-Report, ECN-C-04-087, Augustus 2004.

[11] P.J.M. Heskes, P.M. Rooij, H.E. Oldenkamp: "Photovoltaische Inverters and their Behaviour on Poor Power Quality of the Distribution Network " in Dutch: "Photovoltaische Inverters en hun gedrag op matige netkwaliteit". ECN report: ECN-C--03-105, December 2003.

[12] J.F.G. Cobben: "PV and PQ, interaction en limits" (in Dutch: "PV en $\mathrm{PQ}$, interactie en grenzen"), Master Thesis, Technical University Eindhoven, May 2002.

[13] M. Saito, T. Takeshita, N. Matsui, (Nagoya Institute of Technology, Japan) Modeling and Harmonic Suppression for Power Distribution
Systems IEEE Transactions on Industrial Electronics, Vol. 50, No. 6, Dec. 2003.

[14] P.J.M. Heskes (ECN, The Netherlands), J.L. Duarte (TU/e, The Netherlands), "Harmonic reduction as ancillary service by inverters for Distributed Energy Resources (DER) in electricity distribution networks", Cired 2007, May 2007.

[15] H.Akagi, (Tokyo Institute of Technology, Japan), New trends in active power line conditioners, IEEE Transact. on Industry Applications, vol. 32, no. 6, Dec. 1996.

[16] W. R. Ryckaert, K. de Gusseme, D. M. Van de Sype, J. A. Ghijselen, J. A. Melkebeek, (Ghent University, Belgium), Reduction of the Voltage Distortion with a Converter Employed as Shunt Harmonic Impedance, IEEE, 2005.

[17] W.R.A. Ryckaert, J.A.L. Ghijselen, J.A.A. Melkebeek, (Ghent University, Belgium), Optimized Loads for Damping Harmonic Propagation, IEEE, 2002.

\section{AUTHORS' BIOGRAPHIES}

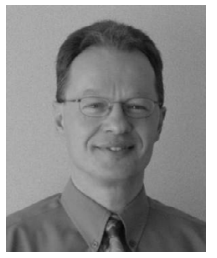

Peter J.M. Heskes received the Electronic Engineer degree from the HTS, The Hague, The Netherlands, in 1980. From 1980 to 1999 , he was with a large Dutch electronic-product manufacturer for the military and professional market. He started there as a Product Designer and became a Product Manager of the powerelectronic department. His work was related to power electronic converters. In 2000, he started as a Project Coordinator with Intelligent Energy Management at the Energy Research Centre of the Netherlands (ECN), Petten, the Netherlands. His current work is related to power electronic converter technology in grid connected distributed energy systems. From the beginning of 2007 he combines his work at ECN with a position as a Ph.D. student at the Electrical Power Systems group of the Eindhoven University of Technology. His research interests are power quality issues with the coupling of power electronic converters of distributed energy systems, to the electricity network.

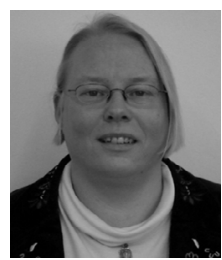

Johanna M.A. Myrzik was born in Darmstadt, Germany in 1966. She received her MSc. in Electrical Engineering from the Darmstadt University of Technology, Germany in 1992. From 1993 to 1995 she worked as a researcher at the Institute for Solar Energy Supply Technology (ISET e.V.) in Kassel, Germany. In 1995 Mrs. Myrzik joined the Kassel University, where she finished her $\mathrm{PhD}$ thesis in the field of solar inverter topologies in 2000. Since 2000, Mrs. Myrzik has been with the Eindhoven University of associate professor in the field of residential electrical infrastructure. In 2009 Johanna Myrzik joined the Technical University of Dortmund where she holds a professorship on energy efficiency. Her fields of interests are: power electronics, renewable energy, distributed generation, electrical power supply.

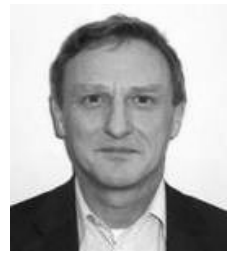

Wil L. Kling (M'95) was born in Heesch, The Netherlands in 1950. He received the M.Sc. degree in electrical engineering from the Eindhoven University of Technology, The Netherlands, in 1978. From 1978 to 1983 he worked with Kema and from 1983 to 1998 with Sep. Since then he is with TenneT, the Dutch Transmission System Operator, as senior engineer for network planning and network strategy. Since 1993 he is a part-time Professor at the Delft University of Technology and since 2000 he is also a part-time Professor in the Electric Power Systems Group at the Eindhoven University of Technology, The Netherlands. From December 2008 he is appointed as a full-time professor and a chair of EPS group at the Eindhoven University of Technology. He is leading research programs on distributed generation, integration of wind power, network concepts and reliability. Mr. Kling is involved in scientific organizations such as Cigre and IEEE. He is the Dutch Representative in the Cigre Study Committee C6, Distribution Systems and Dispersed Generation. 